\title{
The New Information Grey Direct Model and Its Use in The Forecasting of Energy Constitutes
}

\author{
Baohua Yang ${ }^{1, *}$ and Jinshuai Zhao ${ }^{2}$ \\ ${ }^{1}$ Business school, Jiangsu Normal University, Xuzhou, China \\ ${ }^{2}$ School of Computer Science and Technolo, Jiangsu Normal University, Xuzhou, China \\ Corresponding Email: mathyang@126.com
}

\begin{abstract}
To overcome the contradiction of the traditional grey first order accumulate with the new information priority principle, this paper establish the new grey fractional order accumulate, which is proposed to better reflect the priority of the new information. Based on this, This paper establish the fractional order grey direct model based on the principle of new information priority, which is proposed to improve the prediction precision. The results of practical numerical examples demonstrate that this new method has a good predication performance for small data set forecasting. This research also provides a new model for the less data forecasting.
\end{abstract}

\section{Introduction}

Time series prediction refers to the process by which the future values of a system is forecasted based on the information obtained from the past and current data points [1]. $\mathrm{GM}(1,1)$ was first proposed by Deng [2-3], which is one of the most important parts in grey system theory, for small sample forecasting. Because the grey prediction model needs little origin data, has simple calculate process and higher forecasting accuracy, it has been successfully applied in many disciplines [4-7].

The purpose of GM $(1,1)$ model is to work on system forecasting with poor, incomplete or uncertain messages. However, the existing GM $(1,1)$ model cannot be used for accurate prediction for many actual systems, while the system behaviors are affected more or less by other relative factors and their characteristic values never follow the Grey exponential law completely [8]. So, A large number of studies on improved Grey model and applications have been reported, such as Li et al. [9], Xie and Liu [10], Yang et al. [11]. Those improved Grey models all integrated traditional GM $(1,1)$ model and other modeling technology, but not including the research on accumulated generating operator of Grey model.

Although grey forecasting model had been widely adopted, its predicting performance still could to be improved [915]. However, most of improved methods do not emphasize the value of new data, which has been put forwarded in the grey system by Deng, and named the new information priority principle. Dang and Luo improved GM $(1,1)$ using the nth item of $\mathrm{X}(0)(\mathrm{n})$ as the starting condition of the Grey differential model to increase prediction precision [12-15]. Because of the new data represents the future law of development of small sample system [2], we should considered more priority of the nth data than the $\mathrm{n}-1$ th data in the model. Similarly, the 2 th data is more prior than the 1 th data.

Recently, fractional order [16,17] has been applied on the grey generating function, which uses fractional order accumulative generation to narrow the variation of the sequential data and then establish the prediction model. Wu [16] established the grey system model based fractional order accumulative generation by applying the fractional order to grey model. In fact, GM $(1,1)$ model only can simulate homogeneous index sequence, it will has large error when the original data is non-homogenous exponential sequence. So, the grey direct grey model has been formulated to nonhomogenous exponential sequence [18,19]. This paper studies the grey direct grey model based on the technology of fractional order accumulation generation and the principle of priority of new information theory, we establish the optimized model and solve the optimization accumulation order value, then give it in practical application.

The rest of the paper proceeds as follows, the next section presents an overview of the relevant literature on grey model. The third section provides a new fractional order grey direct model with the principle of new information priority of grey system. The fourth section proves the advantage of the grey model put forward in this paper over the traditional grey model by one real case in China. Finally, the paper concludes with some comments in last Section.

\section{Review on $\mathrm{GM}(1,1)$ model and grey direct model}

\subsection{GM(1,1) model [3]}

(C) The Authors, published by EDP Sciences. This is an open access article distributed under the terms of the Creative Commons Attribution License 4.0 (http://creativecommons.org/licenses/by/4.0/). 
Suppose that the non-negative sequence of raw data is $\mathrm{X}(0)=(\mathrm{x}(0)(1), \mathrm{x}(0)(2), \ldots, \mathrm{x}(0)(\mathrm{n}))$, and its sequence of the firstorder accumulative generation operator on $\mathrm{X}(0)$ is denoted as following,

$$
X(1)=(x(1)(1), x(1)(2), \ldots, x(1)(n))
$$

In formula (1), $x^{(1)}(k)=\sum_{i=1}^{k} x^{(0)}(i), \mathrm{k}=1,2, \ldots, \mathrm{n}$

From the sequence $X(1)=(x(1)(1), x(1)(2), \ldots, x(1)(n))$, we can derive the sequence of generated mean value of consecutive neighbors in the following, $Z(1)=(z(1)(2), \ldots, z(1)(n))$; where

$\mathrm{z}(1)(\mathrm{k})=0.5 \mathrm{x}(1)(\mathrm{k})+0.5 \mathrm{x}(1)(\mathrm{k}-1), \mathrm{k}=2, \ldots, \mathrm{n}$.

Definition 1 For a non-negative sequence of raw data $\mathrm{X}(0)$; $\mathrm{X}(1)$ is a generated sequence with the application of the first-order accumulative generation operator on $X(0)$; $Z(1)$ is a sequence with the application of the generated mean value of consecutive neighbors operator on $\mathrm{X}(1)$, then the following equation

$$
x(0)(k)+a z(1)(k)=b, k=2, \ldots, n .
$$

is a grey differential equation, also called model $\mathrm{GM}(1,1)$.

And the equation

$$
\frac{d x^{(1)}}{d t}+a x^{(1)}=b
$$

is the whitened equation of $\mathrm{GM}(1,1)$. If we let

$$
Y=\left[\begin{array}{c}
x^{(0)}(2) \\
x^{(0)}(3) \\
\vdots \\
x^{(0)}(n)
\end{array}\right], B=\left[\begin{array}{cc}
-z^{(1)}(2) & 1 \\
-z^{(1)}(3) & 1 \\
\vdots & \\
-z^{(1)}(n) & 1
\end{array}\right],
$$

One can use formula (2) to make a least square estimation for parameters $\hat{a}=[a, b]^{T}$,

$$
\hat{a}=\left(B^{T} B\right)^{-1} B^{T} Y
$$

After obtaining parameters $a$ and $b$, use formula (5) to define time response formula and use formula (6) to define reducing value sequence.

$$
\begin{gathered}
\hat{x}^{(1)}(k+1)=\left(x^{(0)}(1)-\frac{b}{a}\right) e^{-a k}+\frac{b}{a}, \quad k=1,2, \cdots, n-1 \\
\hat{x}^{(0)}(k+1)=\left(1-e^{a}\right)\left(x^{(0)}(1)-\frac{b}{a}\right) e^{-a k}, \quad k=1,2, \cdots, n-1
\end{gathered}
$$

Finally, one should make error test. The residual sum of squares is shown as formula (7).

$$
s=\varepsilon^{T} \varepsilon=\sum_{k=1}^{m}\left(x^{(0)}(k)-\hat{x}^{(0)}(k)\right)^{2}
$$

Professor Deng points out that the whitened equation and its corresponding time response function cannot be derived from the $\operatorname{GM}(1,1)$ directly, which are some approximate replacement.

\subsection{Grey direct model [18-20]}

Definition 2 Assume that a non-negative sequence $X(0)=(x(0)(1), x(0)(2), \ldots, x(0)(n))$ is given, then

$$
\hat{x}^{(0)}(k+1)=\beta_{0}+\beta_{1} \hat{x}^{(0)}(k)
$$

is referred to as the original form of the auto-regressive $\operatorname{GM}(1,1)$ model (ARGM $(1,1)$ ), or grey direct model.

Theorem 1 Assumed that $X^{(0)}$ is defined by the Definition 2,

$$
B=\left[\begin{array}{cc}
1 & x^{(0)}(1) \\
1 & x^{(0)}(2) \\
\vdots & \vdots \\
1 & x^{(0)}(n-1)
\end{array}\right], Y=\left[\begin{array}{c}
x^{(0)}(2) \\
x^{(0)}(3) \\
\vdots \\
x^{(0)}(n)
\end{array}\right],
$$


Then the lease squares estimation values are

$$
\hat{\beta}=\left(B^{T} B\right)^{-1} B^{T} Y
$$

Among that $\hat{\beta}=\left(\beta_{0}, \beta_{1}\right)^{T}$ is the parameters list of the formula (8).

Theorem 2 Assume that $B 、 Y$ and $\hat{\beta}$ are defined by the Theorem 1 , and let $\hat{x}^{(0)}(1)=x^{(0)}(1)$, then

$$
\hat{x}^{(0)}(k+1)=\left[x^{(0)}(1)-\frac{\beta_{0}}{1-\beta_{1}}\right] \beta_{1}^{k}+\frac{\beta_{0}}{1-\beta_{1}}
$$

Literature [18] pointed out that if the original data is monotonous movements bump geometry, then the application of the formula (8) as shown in the simulation data obtained with the same sequence of model geometry; moreover formula (8) model can fully simulate inhomogeneous index sequence.

Because grey auto-regressive method of $\operatorname{GM}(1,1)$ is formulated by using the original data rather than the accumulated generation data, it does not need to inverse accumulated generating. We can see that formula (6) is an exponential model, formula (9) is a non-homogenous exponential model.

\section{Fractional order grey direct model with the new information priority}

\subsection{Fractional order accumulate with the new information priority}

Fractional contains an "in between" thinking, more and more scholars recognized [16,22], The recent studies have shown that we can enhance the accuracy of gray forecast modeling by select the appropriate fractional order [16].

Definition 3 [16]. Assume that $\mathrm{r}=\mathrm{q} / \mathrm{p}$ and $\left\{x_{j}: j=1, \cdots, m\right\}$ which is a nonnegative sequence, are given, then

$$
\sum_{j=1}^{m}{ }^{(r)} x_{j}=\sum_{j=1}^{m} C_{m-j+r-1}^{m-j} x_{j}
$$

is called the $r$ order accumulated, and define

$$
C_{r-1}^{0}=1, C_{m-j+r-1}^{m-j}=\frac{(m-j+r-1)(m-j+r-2) \cdots(r+1) r}{(m-j) !} .
$$

Supposed that $X^{(0)}=\left(x^{(0)}(1), x^{(0)}(2), \ldots, x^{(0)}(n)\right)$ is non-negative sequence, application of gray system accumulate, we can get the $r$ order accumulated generating sequence $X^{(r)}$ of $X^{(0)}$ is $X^{(r)}=\left(x^{(r)}(1), x^{(r)}(2), \ldots, x^{(r)}(n)\right)$, where $x^{(r)}(k)=\sum_{j=1}^{k} C_{k-j+r-1}^{k-j} x^{(0)}(j), \quad k=1, \ldots, n$. Since $X^{(0)}$ is time series data, the acquired time from $x^{(0)}(1)$ to $x^{(0)}(n)$ is decreasing by the view of time, that is to say, $x^{(0)}(1)$ is the earliest data and $x^{(0)}(n)$ is the latest data, so, $x^{(0)}(1)$ may be referred to as the oldest information and $x^{(0)}(n)$ for the newest message.

From the above $r$ order accumulated generating function, we can see that every accumulate contains the oldest data $x^{(0)}(1)$, that is, we have used the oldest data $\mathrm{n}$ times, and as the latest information $x^{(0)}(n)$, is only used once in the $x^{(r)}(n)$ calculation, which is obviously contradicts with the principle of new information priority in grey system theory. In fact, $x^{(0)}(n)$, as the latest information, is the latest incarnation of the historical, and can be better reflect the internal development and trends of the system. The principle of new information priority is the key point of view about information applied in grey systems theory. That is, by applying additional weights on newer information, one can achieve a better effect from grey prediction. Therefore, the latest information should be paying more consideration when we establish the model and get model parameters. Based on this, we will provide a new cumulative method which can reflect the new information's effect in the following.

Definition 4 For $\mathrm{r}=\mathrm{q} / \mathrm{p}$ and non-negative sequence $\mathrm{X}(0)=(\mathrm{x}(0)(1), \mathrm{x}(0)(2), \ldots, \mathrm{x}(0)(\mathrm{n}))$, then, the $r$ order cumulative $\mathrm{X}(\mathrm{r})=(\mathrm{x}(\mathrm{r})(1), \mathrm{x}(\mathrm{r})(2), \ldots, \mathrm{x}(\mathrm{r})(\mathrm{n}))$ is called the fractional accumulated generating with the new information

priority where

$$
x^{(r)}(k)=\sum_{j=k}^{n} C_{n-j+r-1}^{n-j} x^{(0)}(j)
$$

Fractional derivatives accumulate the whole history of the system in weighted form. The larger $r$ of $x(r)(k)$ is, the larger the weight of old data is; the smaller $r$ of $x(r)(k)$ is, the smaller the weight of old data is. Reducing $r$ can reduce the weights of old data, which can put more emphasis on the newer data.

But from the definition 4 , for any $k$, we can see that all new data have been contained in the $x^{(r)}(k)$, and the more new data, the more weight has been putted because of $C_{n-j+r-1}^{n-j}$ is accessioned with $j$ 's increases. So, the text of the accumulated sequence obtained this way is called the accumulation of new information accumulated priority sequence.

\subsection{Fractional order grey direct model with the new information priority}

Definition 5 Assume that a non-negative sequence $X^{(0)}=\left(x^{(0)}(1), x^{(0)}(2), \ldots, x^{(0)}(n)\right)$ is given, then 


$$
\hat{x}_{(0)}^{(r)}(k+1)=\beta_{0} \sum_{j=k}^{n-1} C_{n-1-j+r-1}^{n-1-j}+\beta_{1} \hat{x}_{(0)}^{(r)}(k)
$$

is called $r$ order grey direct model based on the new information priority, where

$x_{(0)}^{(r)}(k)=\sum_{j=k}^{n} C_{n-j+r-1}^{n-j} x^{(0)}(j), k=1,2, \cdots, n-1$.

Formula (11) and formula (8) is the same in the form, in fact, they are different to each other, the difference lies in the way of data generated essentially. The innate character difference makes the new grey model can not only reflect the new information priority principle of gray system, but also has better accuracy than old, and the advantages of the new model will present in the example.In addition, if $r=1$, the new model reduces to the literature [20] given model, that is the model of formula (11) shown in more general.

Theorem 3 Assumed that $X^{(0)}$ and $\hat{x}_{(0)}^{(r)}(k)$ are defined by the Definition 5,

$$
B=\left[\begin{array}{cc}
\sum_{j=1}^{n-1} C_{n-1-j+r-1}^{n-1-j} & \sum_{i=1}^{n} C_{n-i+r-1}^{n-i} x^{(0)}(i) \\
\sum_{j=2}^{n-1} C_{n-1-j+j-1}^{n-1-j} & \sum_{i=2}^{n} C_{n-i+r-1}^{n-i} x^{(0)}(i) \\
\vdots & \vdots \\
\sum_{j=n-1}^{n-1} C_{n-1-j+r-1}^{n-1-j} & \sum_{i=n-1}^{n} C_{n-i+r-1}^{n-i} x^{(0)}(i)
\end{array}\right], \quad Y=\left[\begin{array}{c}
\sum_{i=2}^{n} C_{n-i+r-1}^{n-i} x^{(0)}(i) \\
\sum_{i=3}^{n} C_{n-i+r-1}^{n-i} x^{(0)}(i) \\
\vdots \\
\sum_{i=n}^{n} C_{n-i+r-1}^{n-i} x^{(0)}(i)
\end{array}\right]
$$

Then the lease squares estimation values are

$$
\hat{\beta}=\left(B^{T} B\right)^{-1} B^{T} Y
$$

Among that $\hat{\beta}=\left(\beta_{0}, \beta_{1}\right)^{T}$ is the parameters lists of the formula ( 11$)$.

Theorem 4 Assume that a non-negative sequence $X^{(0)}=\left(x^{(0)}(1), x^{(0)}(2), \ldots, x^{(0)}(n)\right)$ is given. Actually, Let error is $u$; then

$$
x^{(0)}(k+1)=\beta_{0}+\beta_{1} x^{(0)}(k)+u_{k}, k=1, \ldots, n-1
$$

Via the treatment of new information priority, the mean of the error is $E(A u)$ :

Where

$$
E(A u)=u_{n}+\frac{(n-2) C_{n-(n-1)+r-1}^{n-(n-1)}}{n-1} u_{n-1}+\cdots+\frac{2 C_{n+3+r-1}^{n-3}}{n-1} u_{3}+\frac{C_{n-2+r-1}^{n-2}}{n-1} u_{2} ;
$$

$$
A=\left[\begin{array}{ccccc}
C_{n-2+r-1}^{n-2} & C_{n-3+r-1}^{n-3} & \cdots & C_{n-(n-1)+r-1}^{n-(n-1)} & C_{n-n+r-1}^{n-n} \\
0 & C_{n-3}^{n-3}+r-1 & \cdots & C_{n-(n-1)+r-1}^{n-(n-1)} & C_{n-n+1-1}^{n-n} \\
\vdots & \vdots & \vdots & \vdots & \vdots \\
0 & \cdots & 0 & C_{n-(n-1)+r-1}^{n-(n-1)} & C_{n-n+r-1}^{n-n} \\
0 & \cdots & 0 & 0 & C_{n-n+r-1}^{n-n}
\end{array}\right], u=\left[\begin{array}{c}
u_{1} \\
\vdots \\
u_{n-2} \\
u_{n-1}
\end{array}\right]
$$

Proof: Let

$$
Y_{1}=\left[\begin{array}{c}
x^{(0)}(2) \\
\vdots \\
x^{(0)}(n-1) \\
x^{(0)}(n)
\end{array}\right] B_{1}=\left[\begin{array}{cc}
1 & x^{(0)}(1) \\
\vdots & \vdots \\
1 & x^{(0)}(n-2) \\
1 & x^{(0)}(n-1)
\end{array}\right]
$$

Then via the treatment of new information priority, formula (13) becomes

$$
A Y=A B\left[\begin{array}{l}
\beta_{0} \\
\beta_{1}
\end{array}\right]+A \mu
$$

Obviously $E(A u)=u_{n-1}+\frac{(n-2) C_{n-(n-1)+r-1}^{n-(n-1)}}{n-1} u_{n-2}+\cdots+\frac{2 C_{n-3+r-1}^{n-3}}{n-1} u_{2}+\frac{C_{n-2+r-1}^{n-2}}{n-1} u_{1}$ Because of

$C_{n-i+r-1}^{n-i}=\frac{(n-i+r-1)(n-i+r-2) \cdots(r+1) r}{(n-i) !} \leq 1$

So, 


$$
\begin{aligned}
E(A u) & =u_{n-1}+\frac{(n-2) C_{n-(n-1)+r-1}^{n-(n-1)}}{n-1} u_{n-2}+\cdots+\frac{2 C_{n-3}^{n-3}+r-1}{n-1} u_{2}+\frac{C_{n-2+r-1}^{n-2}}{n-1} u_{1} \\
& \leq u_{n-1}+\frac{n-2}{n-1} u_{n-2}+\cdots+\frac{2}{n-1} u_{2}+\frac{1}{n-1} u_{1}
\end{aligned}
$$

That is the average error of the simulation sequence is more small when we consider the fractional order accumulate with the new information priority.

\section{The determine to the fractional order parameter of grey direct model based on the new information priority}

Based on the discussion above, we can see that the fractional cumulative model's average error is smaller, so the new model has better modeling accuracy. Of course, how to choose the accumulated order $r$ is an important issue. A simple and natural way is establish an optimal model to get the cumulative order $r$, such as the average relative error of simulation data sequence to establish minimum target on the cumulative order optimization model. Therefore, we will establish the cumulative order optimization model, the goal is minimization the simulate sequence's average relative percentage error $(A R P E)$.

$$
\begin{aligned}
& \min \text { ARPE } \\
& \text { s.t } \quad \text { ARPE }=\frac{1}{n-1} \sum_{k=1}^{n-1}\left|\hat{x}^{(0)}(k+1)-x^{(0)}(k+1)\right| \\
& \quad \hat{x}^{(0)}(k+1)=\left[x^{(0)}(1)-\frac{\beta_{0}}{1-\beta_{1}}\right] \beta_{1}^{k}+\frac{\beta_{0}}{1-\beta_{1}} \\
& \quad r>0
\end{aligned}
$$

Obviously, the above optimization model (14) is a nonlinear optimization model; we will use the modern intelligent algorithms, such as genetic algorithms, simulated annealing algorithm, PSO et.al, to solve this model. So, in the next computational analysis, we will use the PSO algorithm to get the accumulated order $r$.

PSO(Particle Swarm ,PSO) is a kind bionic optimization algorithm, and the method comes from the simulation of motor behaviour of birds and fish [21].Assumed the objective is a $n$ dimensional function, the size of particle population is $m$, the $i$-th particle's "best" value is $p_{i}^{\text {best }}$, and global "best" value is $p_{g}^{\text {best }}$, then the $i$-th particle modified its velocity and position according to the following formulas:

$$
\left\{\begin{array}{l}
v_{i d}=w \cdot v_{i d}+c_{1} \operatorname{rand}()\left(p_{i d}^{\text {best }}-x_{i d}\right)+c_{2} \operatorname{Rand}()\left(p_{g d}^{\text {best }}-x_{i d}\right) \\
x_{i d}=x_{i d}+v_{i d}
\end{array}\right.
$$

Where $i=1, \ldots, m, d=1, \ldots, n, \operatorname{rand}()$ and $\operatorname{Rand}()$ are two separately random function in the range[0,1], $c_{1}$ and $c_{1}$ are constants known as acceleration coefficients and $c_{1}=c_{1}=2, w$ is the weigh value of the inertia.

Algorithm process of particle swarm optimization:

Step1: Initializing the population size of particles, every particle's velocity and position;

Step2: For every particle, according to the formula(3) to calculate $\hat{\beta}=\left(\beta_{0}, \beta_{1}\right)^{T}$, then according to the formula (4), $\hat{x}^{(0)}(k+1), k=1,2, \ldots, n-1$ are obtained;

Step3: Calculating every particle's fitness value and get $p_{i}^{\text {best }}$ and $p_{g}^{\text {best }}$;

Step4: Updating each particle's velocity $v_{i}$ and position $x_{i}$ according to the formula $(6)$;

Step5: if the stop condition (the iteration number or the error precision satisfy the request) is satisfied, the iteration stop and output the $p_{g}^{\text {best }}$, that is the optimization $r$; otherwise turn to step 2 .

\section{Example:the simulation of natural gas consumption in china}

Energy has an influencing role in achieving economic and social progress. Forecasting energy Constitutes a vital part of energy policy of a country, especially for a developing country like China whose economy is in a stage of energy transition: from low efficiency solid fuels to oil, gas and electric power. A large number of studies on energy consumption forecasting using grey model and improved grey model have been reported, such as $\mathrm{Wu}$ et al [20], Xu et al [23], Li et al [24].

In this section, the advantage of the fractional order grey direct model based on the new information priority over the other grey models is demonstrated by natural gas consumption in china. Average relative percentage error ( $A R P E)$ compares the real and forecasted values to evaluate the precision.

We consider an example from paper [23] which provides the sample data. Using the data from 1995 to 2006 (insample data) to construct fractional order grey direct model based on the new information priority:

$$
x(k)=24.2617 \times 1.268^{k}+141.6864
$$


then predict the actual value from 2007 to 2008 .

Actual values and the forecasting values of three compared models are presented in Table 1. As can be seen from Table 1, fractional order grey direct model based on the new information priority yielded the lowest ARPE compared with the other models across all the period from 1995-2008, which also illustrate that the new model is more suitable for natural gas consumption.

Table 1. The fitted values and ARPE of four grey models.

\begin{tabular}{|c|c|c|c|c|}
\hline \multirow{2}{*}{ Year } & \multicolumn{4}{|c|}{ Natural gas consumption (From China government webpage) } \\
\cline { 2 - 5 } & Actual value & model (15) & GMNI(1,1) [20] & reference [23] \\
\hline 1995 & 177.531 & 172.406 & 173.677 & 190.243 \\
\hline 1996 & 188.049 & 180.655 & 181.593 & 183.658 \\
\hline 1997 & 176.132 & 191.119 & 191.694 & 184.908 \\
\hline 1998 & 218.699 & 204.395 & 204.583 & 193.994 \\
\hline 1999 & 215.337 & 221.231 & 221.030 & 210.914 \\
\hline 2000 & 244.811 & 242.592 & 242.015 & 235.669 \\
\hline 2001 & 274.554 & 269.687 & 268.793 & 268.259 \\
\hline 2002 & 292.180 & 303.058 & 302.961 & 308.685 \\
\hline 2003 & 339.454 & 347.569 & 346.559 & 356.945 \\
\hline 2004 & 397.285 & 402.968 & 402.191 & 413.040 \\
\hline 2005 & 486.936 & 474.128 & 473.177 & 476.970 \\
\hline 2006 & 561.051 & 562.129 & 563.756 & 548.735 \\
\hline ARPE (\%) & - & 3.12 & 3.092 & 4.406 \\
\hline 2007 & 698.903 & 675.029 & 679.333 & 721.62 \\
\hline 2008 & 807.857 & 818.246 & 826.811 & 925.69 \\
\hline ARPE (\%) & - & 2.35 & 2.573 & 10.75 \\
\hline
\end{tabular}

\section{Conclusion}

Based on principle of priority of new information in the grey system theory, this paper establish a new information accumulates method by use of Fractional accumulate ideas; based on this, a new fractional order direct gray model with information priority has been presented, and given the model parameters with least squares estimation; and analyzed the simulation error of the new model. The results show that fractional order gray direct model with the new information priority can reduce simulation error; the application example also show that the new model has better prediction accuracy and prediction accuracy of analog data. Although the proposed model overcomes the defects of parameters estimation in traditional direct grey model, they may be superior to other modeling methods in some aspects. There are some potential drawbacks such as the selection of tradeoff parameter related to a least squares cost function. The performance of this model is more related to the selection of trade-off parameter. Thus, how to quickly and accurately select the model trade-off parameter should be further studied. Further, by calculating, we have found that the prediction error maybe worse when we use the accumulated order, which can be obtained by the minimize averaging the error between the raw data and simulation data. Therefore, in order to get the optimization cumulative order, what evaluation criteria as objective function is suitable is also an important issue for future research process.

\section{Acknowledgement}

This paper is supported by the National Nature Science Fund of China(No.71301064) and the Ministry of Education Humanities and Social Science Foundation of China (No.12YJC630262).

\section{References}

1. E Kayacan, B Ulutas \& O Kaynak Kaynak. Grey system theory-based models in time series prediction, Expert Systems with Applications, 33, 2(2010), 1784-1789.

2. J L Deng. Contral problems of grey system, Systems \& Contral Letters, 1,5(1982), 288- 294.

3. Liu S F, Lin Y. Grey systems Theory and Applications, Berlin: Springer, 2010. 
4. D C Li, C J Chang, C C Chen.et al. Forecasting short-term electricity consumption using the adaptive grey-based approach--An Asian case. Omega, 40,6(2012),767-773.

5. T S Chang, CY Ku, HP Fu. Grey theory analysis of online population and online game industry revenue in Taiwan. Technological Forecasting and Social Change, 80(2013),175-185.

6. M Evans. An alternative approach to estimating the parameters of a generalized grey Verhulst model: An application to steel intensity of use in the UK, Expert Systems with Applications, 41 (2014), 1236-1244.

7. W Yan, C Y Yu, R Tu, et al. Fire detection model in Tibet based on Grey-fuzzy neural network algorithm. Expert Syst Appl, 38,8(2011),9580-9585.

8. 8.T Li. A research on the Grey prediction model GM(1,n). Appl Math Comput , 218,1(2012),4903-49018.

9. 9.D C Li, C J Chang, C C Chen. et al. Forecasting short-term electricity consumption using the adaptive Greybased approach-an Asian case. Omega,40,6(2012),767-773.

10. N M Xie, S F Liu. Discrete Grey forecasting model and its optimization. App Math Model,33(2009),1173 - 86.

11. B H Yang, Z G Fang, K Zhang . Discrete GM(1,1) model based on sequence of stepwise ratio. Journal of Systems Engineering and Electronics, 34,4 (2012), 715-718.

12. $\mathrm{T} X$ Yao, $\mathrm{S} F$ Liu, $\mathrm{N}$ M Xie. On the properties of small sample of $\mathrm{GM}(1,1)$ model. App Math Model ,33(2009),1894- 903.

13. Y G Dang, S F Liu, K J Chen. The GM models that be taken as initial value. Kybernetes,33,2(2004),247 - 54.

14. Y X Luo. Non-equidistant step by step optimum new information GM(1,1) and its application. Syst Eng Theor Pract,30,12(2010),2254-8.

15. Y H Wang, Y G Dang, Y Q Li, et al. An approach to increase prediction precision of GM(1,1) model based on optimization of the initial condition. Expert Syst Appl ,37,8 (2010),5640-5643.

16. L F Wu, S F Liu, L G Yao, et.al. Grey system model with the fractional order accumulation. Commun Nonlinear Sci Numer Simulat,18(2013),1775-1785.

17. L F Wu, S F Liu, L G Yao. Discrete grey model based on fractional order accumulate, Systems Engineering-Theory \& Practice,34,7(2014),1882-1887.

18. B Zeng, S F Liu. Direct modeling approach of DGM $(1,1)$ with approximate non-homogeneous exponential sequence. Systems Engineering--Theory \& Practice, 31,2(2014),297 -301.

19. Y N Wang. GM $(1,1)$ direct modelling method and its characteristic. Systems Engineering--Theory \& Practice, 8,1(1998):27 -31.

20. L FWu, S F Liu, H J Chen,et..al. Using a novel grey system model to forecast natural gas consumption in China, Mathematical Problems in Engineering,2015

21. J Kenned, R Eberhart. Particle swarm optimization, In: Proc IEEE Int Conf on Neural Networks,1995,1942-1946.

22. R Almeida, A B Malinowska. Generalized transversality conditions in fractional calculus of variations, Communications in Nonlinear Science and Numerical Simulation, 18,3(2013), 443-452.

23. G Xu, W G Wang. Forecasting China's natural gas consumption based on a combination model, Journal of Natural Gas Chemistry,19(2010):493-496.

24. J C Li, X C Dong, M Hook .et.al. Forecasting the growth of China's natural gas consumption, Energy, $36,3(2011), 1380-1385$. 